\title{
Patient-specific instrument assisted calcaneal fracture minimally invasive internal fixation is accurate and practical: a retrospective study
}

\section{Chenggong Wang}

Xiangya Hospital Central South University

\section{Can Xu}

Xiangya Hospital Central South University

\section{Mingqing Li}

Xiangya Hospital Central South University

\section{Hui Li}

Xiangya Hospital Central South University

\section{Han Xiao}

Xiangya Hospital Central South University

\section{Da Zhong}

Xiangya Hospital Central South University

Hua Liu ( $\nabla$ dr_liuhua_xyyy@126.com )

Xiangya Hospital Central South University https://orcid.org/0000-0003-2034-6407

\section{Research article}

Keywords: Whole process-assisted surgical technique, Digital surgical simulation, Patient-specific instrument, Calcaneal fracture, Minimally invasive internal fixation

Posted Date: January 23rd, 2020

DOl: https://doi.org/10.21203/rs.2.21684/v1

License: (c) (i) This work is licensed under a Creative Commons Attribution 4.0 International License. Read Full License 


\section{Abstract}

Background: Almost all of the traditional calcaneal fracture minimally invasive internal fixation (MIIF) require a lot of intraoperative fluoroscopy, moreover, the fracture recovery of many patients are not considered ideal. Therefore, we designed a new surgical procedure through the digital surgical simulation and made patient-specific instrument (PSI) for calcaneal fracture patients before surgery, and then we manage the operation through the assistance of PSI during the whole process. The purpose of this study is to verify whether the PSI assisted calcaneal fracture minimally invasive internal fixation is accurate and practical.

Methods: We retrospectively analyzed the Sanders type III or IV fresh calcaneal fracture patients who had been performed MIIF assisted by the PSI in our hospital during January 2016 to December 2018. We collected and analyzed perioperative data including intraoperative fluoroscopy times, whether the internal fixation actual usage (IFAU) same as preoperative plan, surgical time and situation of complication; and collected and analyzed the data of preoperative actual measurement, preoperative-plan and postoperative actual measurement from X-rays and CT, including Böhler angle, Gissane angle, subtalar joint width, calcaneus valgus angle and calcaneal volume overlapping ratio. All patients had been followed up and their AOFAS score had been recorded. Then we performed statistical analyses.

Results: The intraoperative fluoroscopy times was $3.95 \pm 1.78$, the condition of IFAU in 16 patients was the same as preoperative plan, the surgery time was $28.16 \pm 10.70 \mathrm{~min}$, no patients developed complications. The preoperative plan Böhler angle, Gissane angle, subtalar joint width and calcaneus valgus angle were not significant different from the postoperative; the preoperative Böhler angle, Gissane angle, subtalar joint width and calcaneus valgus angle were all significant different from the postoperative. The calcaneal volume overlapping ratio with preoperative design was $91.2 \pm 2.3 \%$. The AOFAS score of all patients gradually increased as time goes on, and difference of the AOFAS scores between all time-points were significant.

Conclusions: The new PSI can indeed carry out the preoperative plan accurately and quickly, and the PSI assisted calcaneal fracture minimally invasive internal fixation is accurate and practical.

\section{Background}

Currently, more doctors prefer to use minimally invasive surgery to treat fresh closed calcaneal fracture [1, 2]. In this procedure, the surgeon's often reduce fracture by closed reduction or open reduction with a small incision, and the surgeon's often fix fracture by percutaneous fixation [3]. This minimally invasive approach has many advantages compared with the previous open reduction Internal fixation (ORIF)[4,5], such as less incision complications[6], shorter time of limb swelling[7], faster recovery[8], lower rate of internal fixation failure[9], etc.

The classical minimally invasive surgical method for calcaneal fractures includes fracture reduction, temporary fixation and internal fixation implantation. However, the surgical procedure still has some 
disadvantages: Firstly, almost all of the above surgical procedures require a lot of intraoperative fluoroscopy, because the surgeons need to confirm the fracture and internal fixation by using fluoroscopy repeatedly to determine the procedure at each step of the operation [10]. Secondly, the fracture recovery and internal fixation of many patients are not considered ideal at follow-up, because the surgeons determine the procedure at each step of the operation all depend on the surgeon's experiences and are often not optimal [11].

Many studies have demonstrated that digital surgical simulation combines whole process-assisted surgical technique can optimize the operation process and make surgery personalized and precision [12]. In recent years, with the gradual application of three-dimensional printing technology and patient-specific instrument (PSI) in surgery, it has provided many good references for orthopedic surgery [13], in addition, based on years of experience in calcaneus biomechanical study and using digital orthopedic technology such as PSI assisted surgery of our team [14-16], we intends to change the classical calcaneal fracture minimally invasive internal fixation from traditional "focusing on internal conditions" to new "focusing on external auxiliary tools", so as to optimize the surgery into a new surgical method that is more directly, faster and more accurate.

To solve this problem, we designed a new surgical procedure through the digital surgical simulation and made PSI for calcaneal fracture patients before surgery, and then we manage the operation through the assistance of PSI during the whole process. The whole process of the operation assisted by PSI, and performed the preoperative plan step-by-step, which is different from the traditional surgery method fundamentally. The technology, which has been validated by numerous model trials and approved by ethics committees, has been used in a clinical study in our hospital. Therefore, we conducted a retrospective study, which purpose is to verify whether this new PSI can carry out the preoperative plan accurately and quickly, and can help the completion of calcaneal fracture minimally invasive internal fixation accurately and practically.

\section{Methods}

\section{Patients}

This study has been approved by the Ethics Committee of our hospital. We retrospectively analyzed the data of Sanders type III or IV fresh calcaneal fracture patients who had been performed calcaneal fracture minimally invasive internal fixation (MIIF) during January 2016 to December 2018 in our hospital. Data were obtained from the registration system of foot and ankle surgery department, the medical record information system and the follow-up system of patients' service center of our hospital. All patients included in this retrospective study were all agreed to participate in the study and have been signed written consent. All cases were operated by professor Liu. H, who has more than 150 surgical experience of calcaneal fracture internal fixation. The inclusion criteria were: 1) fresh calcaneal fracture; 2) Sanders type III or IV; 3) had been treated by surgery by using PSI-assisted; 4) voluntary provision of medical records to this study. The exclusion criteria were as follows: 1 ) refused to participate in this study; 2 ) the 
homolateral lower limb includes other operations; 3) physical activity disorders caused by other disease, such as stroke; 4) mental illness. By carefully searching the system with our study design, 19 patients (20 feet) were enrolled in our study and received follow-up. Subjects includes 11 males and 8 females with the average age of $37.90 \pm 13.63$ years (range 19 to 65 years old). All patients are diagnosed with Sanders III or IV fresh calcaneal fracture by an experienced orthopedic surgeon through standard CT scanning. 7 patients were diagnosed with Sanders III and 13 with Sanders IV. 12 patients were diagnosed with left calcaneal fracture, 6 with right and 1 with bilateral. All subjects underwent X-ray and CT scanning before their operations. (Table 1).

\section{Preoperative planning}

The CT images of the patient's foot had been obtained with a Philips scanner (Philips Medical Systems, Eindhoven, Netherlands) at 0.6-mm slice thickness and converted to the DICOM format. A 3-dimensional model of the calcaneus was created with Mimics 19.0 software (Materialise, Leuven, Belgium). We found that the calcaneus had split into a number of main parts (usually 3 to 5 parts). We used the software to mimic anatomic reduction of the calcaneus and then generated the PSI, which had 2 parts in the following steps: a) used 3 to 5 Schanz pins (or K-wires) to independently connected the fragments which would be used for the reduction of the fracture as hand grips; b) simulated reduction of the calcaneus in the digital model, then made sure how to fix the fracture and get the screws' size, orientation information. The simulation allowed us to understand that we needed to insert 1 cortical screw from outside of the left foot to inside beneath the posterior subtalar joint and then inserted 1 lag screw from the margin of the attachment of the Achilles tendon to the calcaneocuboid joint. Insertion of another lag screw from a point 1 to $2 \mathrm{~cm}$ below the insertion point of the first lag screw to the middle subtalar joint was needed. c) designed the PSI part 1- a instrument used to reduce and immobilize the calcaneus with the K-wires going through it and guide the orientation of the screws. d) got back to the original state with the Schanz pins connecting to the fragments, designed the PSI part 2 that used to mark the position of the Schanz pins (or K-wires) (Figure 1). Finally, based on the digital information, a PSI was generated. We chose nylon material to make the PSI by Selective Laser Sintering (SLS) technique.

\section{Surgery}

The patient was placed in the supine position. under nerve blocking anesthesia, and was tourniqueted at the upper thigh. The surgery was a reverse process of the procedure of designing the PSI. Surgical procedures are divided into the following 7 steps (Figure 2):

1. We exposed surgical area.

2. We installed the PSI Part 2 to his left foot and ankle, the function of Part 2 is a guide used to guide the installation of reset Schanz pins (or K-wires).

3. We inserted 3 to 5 Schanz pins (or K-wires) to independently connect the fragments through the Schanz pin holes of PSI Part 2. (Figure 3-A): 
4. We removed PSI Part 2 with schanz pins connecting to the calcaneus and were ready to reduce the calcaneus. At this point, each Schanz pin (or K-wires) forms a rigid complex with the fracture fragments.

5. We grabbed the Schanz pins (or K-wires) to preliminary reduce the calcaneus guided by our understanding of the characteristics of the displaced fracture. At the same time, the PSI Part 1 was assembled with the Schanz pins (or K-wires) gone through the Schanz pin holes (or K-wire holes) (Figure 3-B). Ideal anatomical reduction was achieved once the PSI Part 1 was fixed on the foot with Schanz pins (or K-wires) pushed through the Schanz pin holes (or K-wire holes), because the function of Part 1 defining the position of the Schanz-pin-fragments rigid complex (or K-wire-fragments rigid complex).

6. We placed a 8 to $20 \mathrm{~mm}$ wide incision on the lateral of subtalar joint, and implant one or two cortical screws to fix the subtalar articular surface by using part 1's internal fixation implant guide kit. (Figure 3-C)

7. We implant two or three cannulated screws to fix the main body of the calcaneus axially by using part 1's internal fixation implant guide kit. (Figure 3-D)

After all internal fixations have been installed, we performed a C-arm check. Finally, we disassembled the PSI and took out the Schanz pins (or K-wires).

\section{Postoperative Management}

After surgery, all patients were required to raise the limb, and the use of antibiotics was necessary. All patients wore a plaster slab until the stitch of wound was removed immediately after surgery, and then wore a supramalleolar protective plaster cast. Patients were encouraged to mobilize non-weight bearing for the first 3-8 weeks, partially weight bearing for several weeks according to the doctor's advice, and full weight bearing thereafter. The majority of plaster casts of patients were removed between 6 and 8 weeks after surgery.

\section{Measurement and follow-up}

We carefully searched and collected the following data through the medical records and follow-up system: Intraoperative fluoroscopy times, whether the internal fixation actual usage (IFAU) same as preoperative plan, surgical time and situation of complication; and then, we collected the data of preoperative actual measurement, preoperative-plan and postoperative actual measurement from standard radiological examinations (X-rays, CT). We recorded the Böhler angle and Gissane angle from Xrays (6 months to 12 months after surgery) (Figure 4). The postoperative CT (6 months to 12 months after surgery) data were imported into the computer and reconstructed to obtain the "postoperative 3D digital model" and compared with the preoperative data. We were going to focus on the situation of the calcaneal shape and anatomical structure, and recorded the subtalar joint width (in the position of sustentaculum) and calcaneus valgus angle (Figure 5). We overlapped the CT 3-dimensional reconstruction calcaneal model after surgery with the ideal calcaneal model designed before surgery, and 
then we calculated the calcaneal volume overlapping ratio (internal fixation has been excluded) (Figure 6). All patients had been followed up, their function score (the American Orthopedic Foot and Ankle Society score, AOFAS score) $[17,18]$ will be recorded at 12 weeks, 6 months and 1 year after surgery. We got these data through outpatient or visiting services.

\section{Statistical analysis}

Data were presented as mean with ranges. Paired samples t- test was used to analyze pre-op, pre-op plan and post-op data. Statistical analyses were conducted by SPSS 22.0 software (SPSS Inc., Chicago, IL, USA). $P$ value less than 0.05 was considered statistically significant.

\section{Results}

One patient received bilateral surgery. The intraoperative fluoroscopy times was $3.95 \pm 1.78$ (range, 2 to 9); The condition of internal fixation actual usage in 16 patients was the same as preoperative plan, but in 3 was not the same as preoperative plan; The surgery time was $28.16 \pm 10.70 \mathrm{~min}$ (range 15 to $50 \mathrm{~min}$ ); No patients developed complications. (Table 2)

The preoperative plan Böhler angle was $31.5 \pm 2.7^{\circ}$ (range, $28^{\circ}$ to $35^{\circ}$ ), the postoperative Böhler angle was $30.2 \pm 5.9^{\circ}$ (range, $20.6^{\circ}$ to $37.7^{\circ}$ ), the different value $5.7 \pm 3.3^{\circ}$ (range, $0.7^{\circ}$ to $12.2^{\circ}$ ) had no significant difference $(P=0.407)$; However, the preoperative actual measurement Böhler angle was $9.6 \pm 6.5^{\circ}$ (range, $-3.1^{\circ}$ to $18.4^{\circ}$ ), which was significant different from the postoperative Böhler angle $20.7 \pm 7.0^{\circ}$ (range, $9.3^{\circ}$ to $\left.36.8^{\circ}\right)(P=0.000)$. The preoperative plan Gissane angle was $125.6 \pm 4.0^{\circ}$ (range, $118^{\circ}$ to $134^{\circ}$ ), the postoperative Gissane angle was $123.6 \pm 4.4^{\circ}$ (range, $115.8^{\circ}$ to $128.7^{\circ}$ ), the different value $5.0 \pm 4.3^{\circ}$ (range, $1.0^{\circ}$ to $\left.17.0^{\circ}\right)$ had no significant difference $(P=0.187)$; However, the preoperative actual measurement Gissane angle was $151.3 \pm 8.0^{\circ}$ (range, $138.2^{\circ}$ to $161.5^{\circ}$ ), which was significant different from the postoperative Gissane angle $27.7 \pm 7.7^{\circ}\left(14.0^{\circ}\right.$ to $\left.41.5^{\circ}\right)(P=0.000)$. The preoperative plan subtalar joint width (in the position of sustentaculum) was $46.8 \pm 1.3 \mathrm{~mm}$ (range, $44 \mathrm{~mm}$ to $48 \mathrm{~mm}$ ), the postoperative subtalar joint width was $46.4 \pm 2.9 \mathrm{~mm}$ (range, $42.5 \mathrm{~mm}$ to $51.9 \mathrm{~mm}$ ), the different value $3.2 \pm 2.0 \mathrm{~mm}$ (range, $0.1 \mathrm{~mm}$ to $7.9 \mathrm{~mm}$ ) had no significant difference $(\mathrm{P}=0.718)$; However, the preoperative actual measurement subtalar joint width was $55.4 \pm 2.8 \mathrm{~mm}$ (range, $50.2 \mathrm{~mm}$ to $59.6 \mathrm{~mm}$ ), which was significant different from the postoperative subtalar joint width $9.0 \pm 4.3 \mathrm{~mm}$ (range, $2.4 \mathrm{~mm}$ to $16.4 \mathrm{~mm})(P=0.000)$. The preoperative plan calcaneus valgus angle was $3.6 \pm 0.8^{\circ}$ (range, $3^{\circ}$ to $5^{\circ}$ ), the postoperative calcaneus valgus angle was $3.3 \pm 1.3^{\circ}$ (range, $1.2^{\circ}$ to $5.6^{\circ}$ ), the different value $1.4 \pm 0.9^{\circ}$ (range, $0.3^{\circ}$ to $3.6^{\circ}$ ) had no significant difference $(\mathrm{P}=0.541)$; However, the preoperative actual measurement calcaneus valgus angle was $-0.1 \pm 5.0^{\circ}$ (range, $-7.5^{\circ}$ to $10.9^{\circ}$ ), which was significant different from the postoperative calcaneus valgus angle $4.6 \pm 1.9^{\circ}$ (range, $-5.3^{\circ}$ to $\left.8.7^{\circ}\right)(P=0.010)$. The calcaneal volume overlapping ratio with preoperative design was $91.2 \pm 2.3 \%$ (range, $87.0 \%$ to $95.8 \%$ ). (Table 3, Figure 7 )

All patients completed three follow-up postoperatively, the AOFAS score of all patients gradually increased as time goes on. The preoperative AOFAS score was $13.1 \pm 4.7$ (range, 5 to 18 ), the postoperative 8 weeks AOFAS score was $62.5 \pm 4.3$ (range, 55 to 70), the postoperative 6 months AOFAS 
score was $80.8 \pm 7.6$ (range, 71 to 92), the postoperative 1 year AOFAS score was $88.6 \pm 5.5$ (range, 81 to 97). Difference of the AOFAS scores between all time-points were significant $(P<0.001)$. (Figure 8 )

\section{Discussion}

For decades, open reduction and internal fixation performed by an extended extensile lateral approach has been the standard treatment for calcaneal fracture [19]. With this treatment, a certain degree of anatomical reduction is achieved, but the occurrence of serious complications has gained surgeons' attention and resulted in the development of a minimally invasive approach [20]. In recent years, with the efforts of scholars, minimally invasive surgery has become a more effectively surgical method solve calcaneal fractures. Nosewicz et al [21] reported sufficient exposure of complex calcaneal fractures by application of a minimally invasive sinus tarsi approach for anatomic reduction and stable fixation. Wang et al [22] applied a minimally invasive lateral approach in 156 patients and found that the percutaneous leverage, manual compression, and application of anatomic plates and compression bolts to be effective for displaced intra-articular calcaneal fractures, offering a combination of fewer soft tissue complications and good reduction.

But the classical minimally invasive surgical method for calcaneal fractures still has some disadvantages. The classical method includes fracture reduction, temporary fixation and internal fixation implantation. On the one hand, almost all of the above surgical procedures require a lot of intraoperative fluoroscopy. On the other hand, the surgeons determine the procedure at each step of the operation all depend on the surgeon's experiences and are often not optimal.

The minimally invasive surgery varies according to the fracture pattern. This makes personalized surgical planning important [23]. With the help of CT imaging and a rapidly produced prototype, surgeons can obtain detailed information regarding the fracture and use software to plan a procedure that will result in suitable fixation [24]. More importantly, the surgery can be simulated in vitro. Although preoperative planning is often near-perfect, it is not possible to fully anticipate the surgical challenges of individual cases. The calcaneus fracture type is complex [25], and there is no single treatment protocol that covers the various kinds of calcaneal fracture. The core point of the PSI was to guide the surgery as planned. we digitally reconstructed the calcaneus on the computer so that we could understand the characteristics of the fracture [26], second, simulating the reduction and fixation, then creating a PSI and using it to guide the actual surgery.

Our team intends to optimize the classical calcaneal fracture minimally invasive internal fixation into a more personalized and precision operation method. Therefore, we have creatively designed an optimal surgical procedure through the digital surgical simulation, and then we manage the operation through the assistance of PSI during the whole process. Combined with the cases in this paper, we preliminarily believe this new surgery method is great different from the classical minimally invasive calcaneal fractures surgery. 
What is unique about this new case we reported is that the traditional calcaneal fracture minimally invasive internal fixation procedure was changed to: preoperative digital surgical simulation and preparation of PSI, install Schanz pins (or K-wires) by using PSI, adjustment of the relationship between Schanz pins (or K-wires) and PSI according to the surgical plan, internal fixation implantation by using PSI. In fact, we changed the operation procedure from traditional "focusing on internal conditions" to new "focusing on external auxiliary tools", so as to optimize the surgery into a new surgical method that is more directly, faster and more accurate.

This paper not only shows a new PSI, but also shows an unprecedented new method for calcaneal fracture internal fixation. In other words, the whole process of the operation assisted by PSI, and performed the preoperative plan step-by-step, which is different from the traditional surgery method fundamentally. In fact, The steps of traditional method are improvisational, surgical procedures have some uncertainty. However, our new method of this study is standardized and relatively step-by-step. In addition, our new method is also very different from the traditional PSI method, which only optimizes a part of procedure, while our new method can improve the whole operation process, or even make transformation of the operation. Based on this case, we found that the new operation method was very simple and the surgery process was smooth, the operation time was less than half of the classic operation, and the postoperative effect was also very good. With the assisted by PSI in whole process, the operation was guided at every step, surgical procedures became routine, intraoperative fluoroscopy was no longer required at every step, fracture reduction and internal fixation became relatively perfect. Therefore, it can be preliminarily believed that such a new type of technology will be expected to optimize the traditional surgical scheme greatly.

Strengths and limitations of the study

Still, there are some limitations of this new method. First, designing the PSI is a complex procedure [27, 28]. Though it doesn't take much time to make it, the surgeon needs a technologist who is skilled at the Mimics software to assist to make the PSI. If the surgeon is good at the software, that won't be a problem. Second, the system also cannot be used to manage an old fracture. We just use the Schanz pins (or K-wires) to reduce fracture, fresh fracture is easy to reduce while the old fracture can't be reduced with closed reduction. Typically, we think it is best to conduct the PSI assisted surgery within 8 hours after injured. Third, this is preliminary application with the small number of sample sizes and short period of follow-up. Therefore, further prospective investigations with the large numbers of sample sizes and longer follow-up duration will be necessary to investigate appropriate values for clinical application.

\section{Conclusion}

This study demonstrated that the surgery procedure of calcaneal fracture minimally invasive internal fixation can be changed from traditional "focusing on internal conditions" to new "focusing on external auxiliary tools". The new PSI can indeed carry out the preoperative plan accurately and quickly, and the PSI assisted calcaneal fracture minimally invasive internal fixation is accurate and practical. 


\section{Abbreviations}

MIIF: Minimally invasive internal fixation; PSI: Patient-specific instrument; ORIF: Open reduction Internal fixation; IFAU: Internal fixation actual usage; SLS: Selected laser sintering; C-arm: C-arm fluoroscopy; AOFAS: American Orthopedic Foot and Ankle Society.

\section{Declarations}

\section{Acknowledgements}

This study was strongly supported by the Digital Research Institute of Orthopaedics of Xiangya Hospital, and we thanks to the technical support of Xiaopeng Wang specially.

\section{Ethics approval and consent to participate}

This study has been approved by the ethics committee of our hospital (No.201902011). All procedures performed in studies were in accordance with the ethical standards of our institutional ethical committee. Informed consent was obtained from all individual participants included in the study.

\section{Consent for publication}

All patients involved had given informed consent.

\section{Competing interests}

The authors declare that they have no competing interests.

\section{Authors' contributions}

Liu.H and Wang.CG contributed to the investigation, methodology, data curation, and preparation of the original draft. Wang.CG contributed to the formal analysis, data curation, and preparation of the original draft. Xiao.H contributed to the data curation and preparation of the original draft. Xu.C and Wang.CG prepared the original draft. Zhong.D and Li.MQ did the validation of data. Li.H and Wang.CG contributed to the design of the PSI. Zhong.D and Liu.H contributed to the surgical guidance. Liu.H and Wang.CG edited and reviewed the manuscript. All authors read and approved the final manuscript.

\section{References}

1. Shih JT, Kuo CL, Yeh TT, Shen HC, Pan RY, Wu CC. Modified Essex-Lopresti procedure with percutaneous calcaneoplasty for comminuted intra-articular calcaneal fractures: a retrospective case analysis. BMC Musculoskelet Disord. 2018;19:77.

2. Yao H, Liang T, Xu Y, Hou G, Lv L, Zhang J. Sinus tarsi approach versus extensile lateral approach for displaced intra-articular calcaneal fracture: a meta-analysis of current evidence base. J Orthop Surg Res. 2017;12:43. 
3. Peng Y, Liu J, Zhang G, Ji X, Zhang W, Zhang L, et al. Reduction and functional outcome of open reduction plate fixation versus minimally invasive reduction with percutaneous screw fixation for displaced calcaneus fracture: a retrospective study. J Orthop Surg Res. 2019;14:124.

4. Humphrey JA, Woods A, Robinson A. The epidemiology and trends in the surgical management of calcaneal fractures in England between 2000 and 2017. Bone Joint J. 2019;101-B:140-6.

5. Zhou HC, Yu T, Ren HY, Li B, Chen K, Zhao YG, et al. Clinical Comparison of Extensile Lateral Approach and Sinus Tarsi Approach Combined with Medial Distraction Technique for Intra-Articular Calcaneal Fractures. Orthop Surg. 2017;9:77-85.

6. Lin J, Xie C, Chen K, Sun S, Zhou K, Zhou C, et al. Comparison of sinus tarsi approach versus extensile lateral approach for displaced intra-articular calcaneal fractures Sanders type IV. Int Orthop. 2019;43:2141-9.

7. Bläsius FM, Link BC, Beeres F, Iselin LD, Leu BM, Gueorguiev B, et al. Impact of surgical procedures on soft tissue microcirculation in calcaneal fractures: A prospective longitudinal cohort study. Injury. 2019;50:2332-8.

8. Cottom JM, Douthett SM, McConnell KK. Intraoperative Reduction Techniques for Surgical Management of Displaced Intra-Articular Calcaneal Fractures. Clin Podiatr Med Surg. 2019;36:26977.

9. Rachakonda KR, Nugur A, Shekar NA, Kidiyur B, Kilaru P, Gannamani S, et al. Minimally invasive fixation for displaced intra-articular fractures of calcaneum: a short-term prospective study on functional and radiological outcome. Musculoskelet Surg. 2019;103:181-9.

10. Feng Y, Shui X, Wang J, Cai L, Yu Y, Ying X, et al. Comparison of percutaneous cannulated screw fixation and calcium sulfate cement grafting versus minimally invasive sinus tarsi approach and plate fixation for displaced intra-articular calcaneal fractures: a prospective randomized controlled trial. BMC Musculoskelet Disord. 2016;17:288.

11. Tomesen T, Biert J, Frölke JP. Treatment of displaced intra-articular calcaneal fractures with closed reduction and percutaneous screw fixation. J Bone Joint Surg Am. 2011;93:920-8.

12. Xia S, Fu B, Wang B, Wu J, Cui Y, Wang X. Computed Tomography Imaging-Based Preoperative Virtual Simulation for Calcaneal Fractures Reduction. J Foot Ankle Surg. 2019;58:248-52.

13. Jones GG, Clarke S, Harris S, Jaere M, Aldalmani T, de Klee P, et al. A novel patient-specific instrument design can deliver robotic level accuracy in unicompartmental knee arthroplasty. Knee. 2019;26:1421-8.

14. Lei PF, Su SL, Kong LY, Wang CG, Zhong D, Hu YH. Mixed Reality Combined with Three-Dimensional Printing Technology in Total Hip Arthroplasty: An Updated Review with a Preliminary Case Presentation. Orthop Surg. 2019;11:914-20.

15. Xu C, Li M, Wang C, Li K, Liu H. RETRACTED: Ankle-hindfoot after calcaneal fractures: A biomechanical study. Orthop Traumatol Surg Res. 2017;103:709-16.

16. Wang C, Xiao H, Yang W, Wang L, Hu Y, Liu H, et al. Accuracy and practicability of a patient-specific guide using acetabular superolateral rim during THA in Crowe II/III DDH patients: a retrospective 
study. J Orthop Surg Res. 2019;14:19.

17. Sanders R, Fortin P, DiPasquale T, Walling A. Operative treatment in 120 displaced intraarticular calcaneal fractures. Results using a prognostic computed tomography scan classification. Clin Orthop Relat Res. 1993;87-95.

18. Kitaoka HB, Alexander IJ, Adelaar RS, A Nunley J, Myerson MS, Sanders M, et al. Clinical Rating Systems for the Ankle-Hindfoot, Midfoot, Hallux, and Lesser Toes. Foot Ankle Int. 1997;18:187-8.

19. Wang C, Huang D, Ma X, Wang X, Huang J, Zhang C, et al. Sustentacular screw placement with guidance during ORIF of calcaneal fracture: an anatomical specimen study. J Orthop Surg Res. 2017;12:78.

20. Chen L, Zhang G, Hong J, Lu X, Yuan W. Comparison of percutaneous screw fixation and calcium sulfate cement grafting versus open treatment of displaced intra-articular calcaneal fractures. Foot Ankle Int. 2011;32:979-85.

21. Nosewicz TL, Knupp M, Bolliger L, Henninger HB, Barg A, Hintermann B. Radiological morphology of peritalar instability in varus and valgus tilted ankles. Foot Ankle Int. 2014;35:453-62.

22. Wang Q, Chen W, Su Y, Pan J, Zhang Q, Peng A, et al. Minimally invasive treatment of calcaneal fracture by percutaneous leverage, anatomical plate, and compression bolts--the clinical evaluation of cohort of 156 patients. J Trauma. 2010;69:1515-22.

23. Takeuchi N, Mae T, Fukushi JI, Tsukamoto N, Mizu-Uchi H, Momii K, et al. Management of IntraArticular Calcaneal Fractures: Clinical Results of Reduction Technique Using a Bone Spreader. J Foot Ankle Surg. 2017;56:1025-30.

24. Yu B, Chen WC, Lee PY, Lin KP, Lin KJ, Tsai CL, et al. Biomechanical comparison of conventional and anatomical calcaneal plates for the treatment of intraarticular calcaneal fractures - a finite element study. Comput Methods Biomech Biomed Engin. 2016;19:1363-70.

25. Xu C, Liu H, Li M, Wang C, Li K. A Three-Dimensional Finite Element Analysis of Displaced IntraArticular Calcaneal Fractures. J Foot Ankle Surg. 2017;56:319-26.

26. Xu C, Zhang MY, Lei GH, Zhang C, Gao SG, Ting W, et al. Biomechanical evaluation of tenodesis reconstruction in ankle with deltoid ligament deficiency: a finite element analysis. Knee Surg Sports Traumatol Arthrosc. 2012;20:1854-62.

27. Evrard R, Schubert T, Paul L, Docquier PL. Resection margins obtained with patient-specific instruments for resecting primary pelvic bone sarcomas: A case-control study. Orthop Traumatol Surg Res. 2019;105:781-7.

28. Sallent A, Ramírez M, Catalá J, Rodríguez-Baeza A, Bagó J, de Albert M, et al. Precision and safety of Multilevel Cervical Transpedicular Screw Fixation with 3D Patient-Specific Guides; A Cadaveric Study. Sci Rep. 2019;9:15686.

\section{Tables}

Table 1 Demographic characteristics of the fresh calcaneal fracture patients in the study. 


\begin{tabular}{|l|l|}
\hline Number of patient & 19 \\
\hline Number of gender (male/female) & $11 / 8$ \\
\hline Mean of age (years) & $37.90 \pm 13.63$ \\
\hline Number of sides (left/right/bilateral) & $12 / 6 / 1$ \\
\hline Number of Sanders classification (III/IV) & $7 / 13$ \\
\hline
\end{tabular}

Table 2 Conditions of surgeries and complications.

\begin{tabular}{|l|l|}
\hline Intraoperative fluoroscopy times & $3.95 \pm 1.78$ \\
\hline IFAU* same as pre-op? (Yes/No) & $16 / 3$ \\
\hline Surgery time (min) & $28.16 \pm 10.70$ \\
\hline Complications & None \\
\hline
\end{tabular}

*IFAU: internal fixation actual usage

Table 3 X-ray and CT 3-dimensional reconstruction data and related comparisons.

\begin{tabular}{|c|c|c|c|c|c|c|c|c|c|}
\hline \multicolumn{2}{|c|}{ Mean } & \multicolumn{4}{|c|}{$\begin{array}{c}\text { Compare preoperative-plan } \\
\text { with postoperative }\end{array}$} & \multicolumn{4}{|c|}{$\begin{array}{c}\text { Compare preoperative actual } \\
\text { measurement } \\
\text { with postoperative } \\
\end{array}$} \\
\hline & & $\begin{array}{l}\text { Pre-op } \\
\text { plan }\end{array}$ & Post-op & $\Delta^{\#}$ & $P *$ & Pre-op & Post-op & $\Delta^{\#}$ & $P^{*}$ \\
\hline \multirow[t]{2}{*}{ ray data } & Böhler angle $\left({ }^{\circ}\right)$ & $31.5 \pm 2.7$ & $30.2 \pm 5.9$ & $5.7 \pm 3.3$ & 0.407 & $9.6 \pm 6.5$ & $30.2 \pm 5.9$ & $20.7 \pm 7.0$ & 0.000 \\
\hline & $\begin{array}{l}\text { Gissane angle } \\
\left({ }^{\circ}\right)\end{array}$ & $125.6 \pm 4.0$ & $123.6 \pm 4.4$ & $5.0 \pm 4.3$ & 0.187 & $151.3 \pm 8.0$ & $123.6 \pm 4.4$ & $27.7 \pm 7.7$ & 0.000 \\
\hline \multirow[t]{2}{*}{$\begin{array}{l}\text { [3- } \\
\text { mensional } \\
\text { construction } \\
\text { ta }\end{array}$} & $\begin{array}{l}\text { Subtalar joint } \\
\text { width } \\
\text { (sustentaculum) } \\
\text { (mm) }\end{array}$ & $46.8 \pm 1.3$ & $46.4 \pm 2.9$ & $3.2 \pm 2.0$ & 0.718 & $55.4 \pm 2.8$ & $46.4 \pm 2.9$ & $9.0 \pm 4.3$ & 0.000 \\
\hline & $\begin{array}{l}\text { Calcaneus } \\
\text { valgus angle }\left(^{\circ}\right)\end{array}$ & $3.6 \pm 0.8$ & $3.3 \pm 1.3$ & $1.4 \pm 0.9$ & 0.541 & $-0.1 \pm 5.0$ & $3.3 \pm 1.3$ & $4.6 \pm 1.9$ & 0.010 \\
\hline
\end{tabular}

*P: P value (paired samples t test)

${ }^{\#} \Delta$ : Different value (absolute value)

Figures 


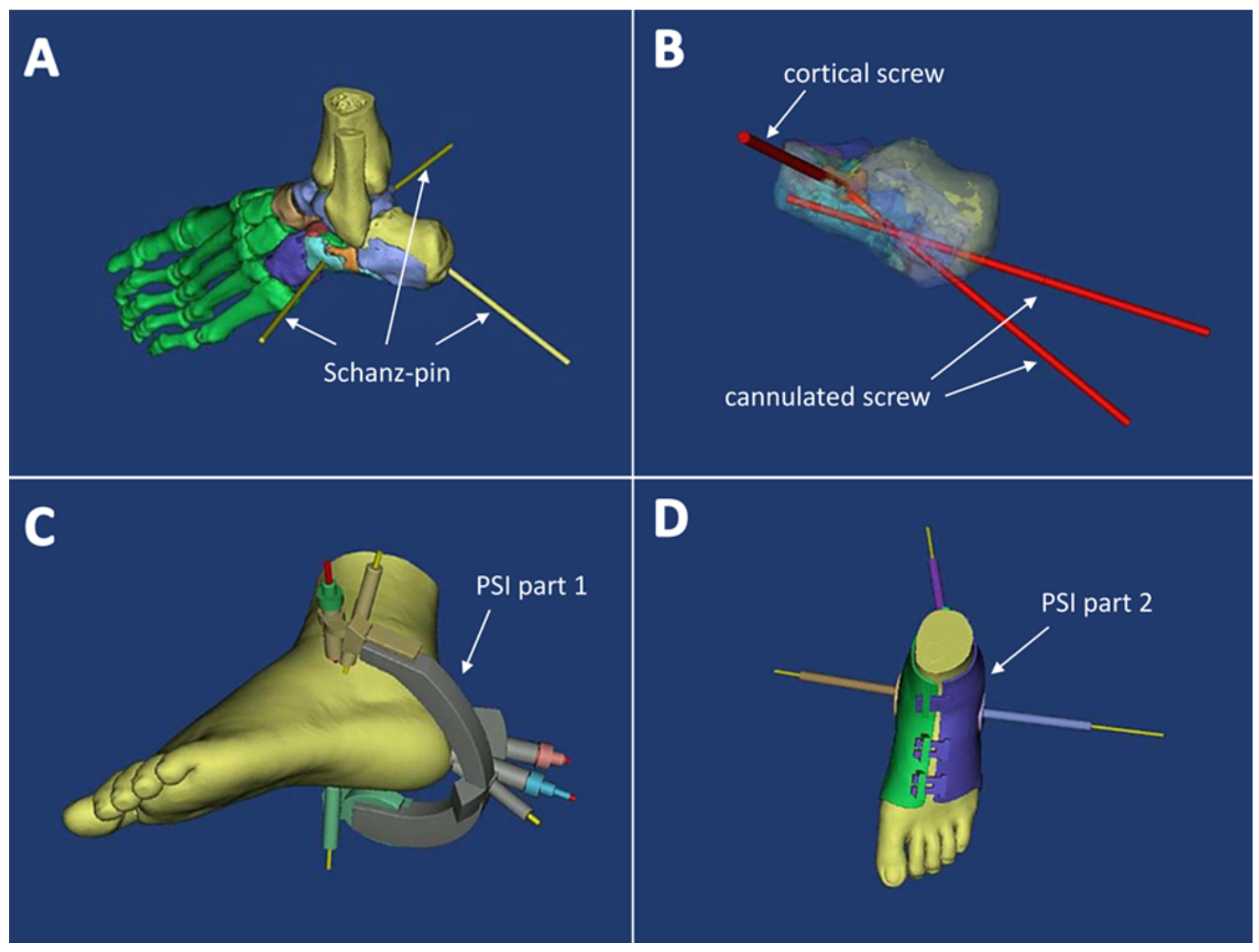

\section{Figure 1}

An example of process of preoperative planning: (A) The location of 3 to 5 Schanz pins (or K-wires) connect fracture fragments was determined by digital simulation. (B) The number, size and location of internal fixation were determined by digital simulation, we make sure each Schanz pin (or K-wire) forms a rigid complex with the fracture fragments. (C) The PSI Part 1-an instrument used to immobilize the calcaneus after reduction and guide the orientation of the screws-is created. (D) The PSI Part 2-which is used to guide placement of the 3 to 5 Schanz pins (or K-wires)-is created for restoration of the calcaneus to its pre-fracture state with Schanz pins (or K-wires) connecting the fragments. 


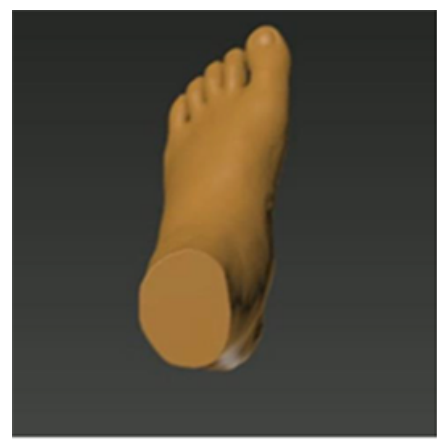

Step 1: Expose surgical area

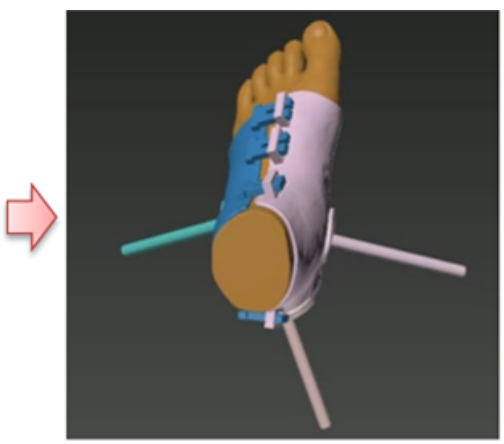

Step 2: Install part 2 (a guide used to guide the installation of reset Schanz pins )

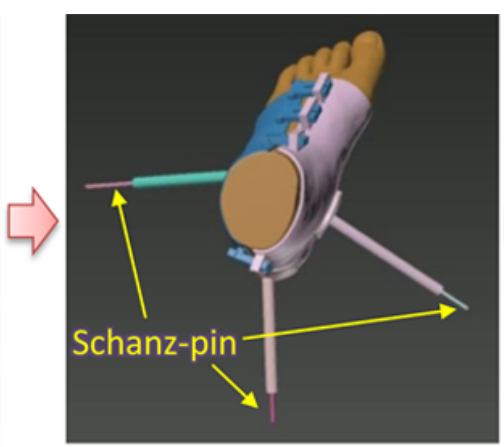

Step 3: Implant 3 Schanz pins using part 2

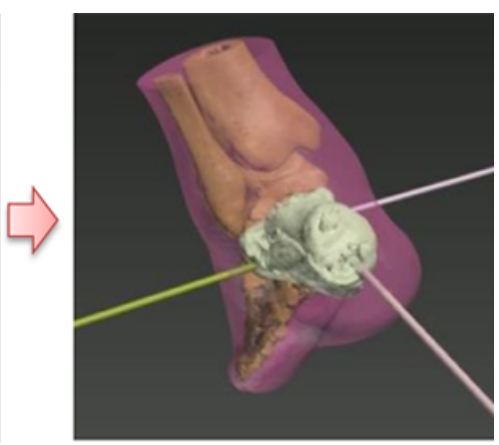

Step 4: Remove part 2 (Now, each Schanz pin forms a rigid complex with the fracture fragments)

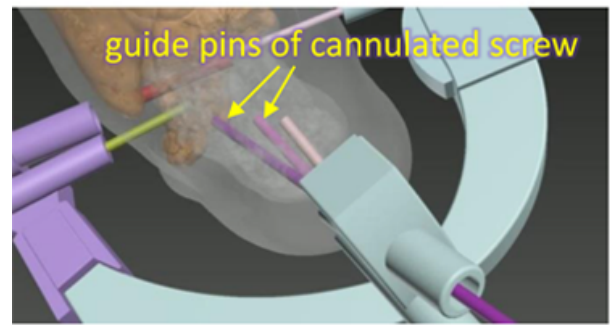

Step 7: Implant two cannulated screws to fix the main body of the calcaneus axially by using part 1 's internal fixation implant guide kit

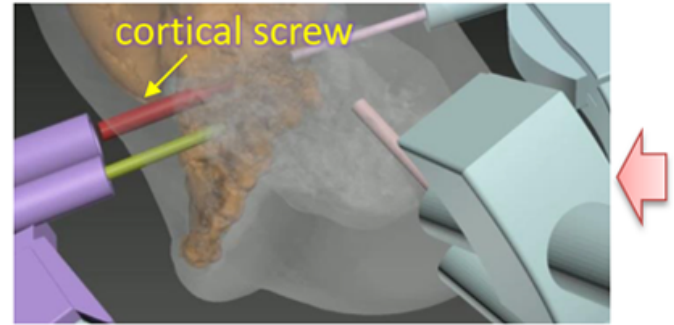

Step 6: Implant one cortical screw to fix the subtalar articular surface by using part 1's internal fixation implant guide kit

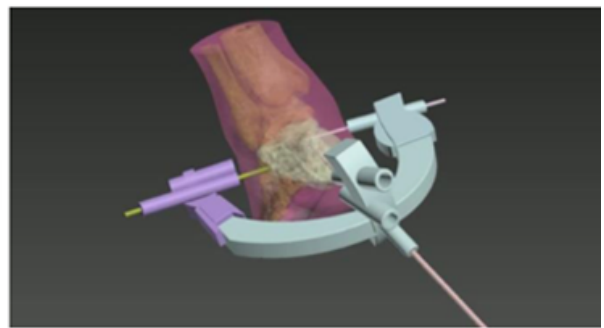

Step 5: Install part 1

(a guide used to reduce the fracture and ensure temporary fixation, by defining the position of the Schanz-pin-fragments rigid complex)

\section{Figure 3}

Schematic diagram of the new surgery method which was divided into 7 steps, PSI assisted operation during the whole process. 


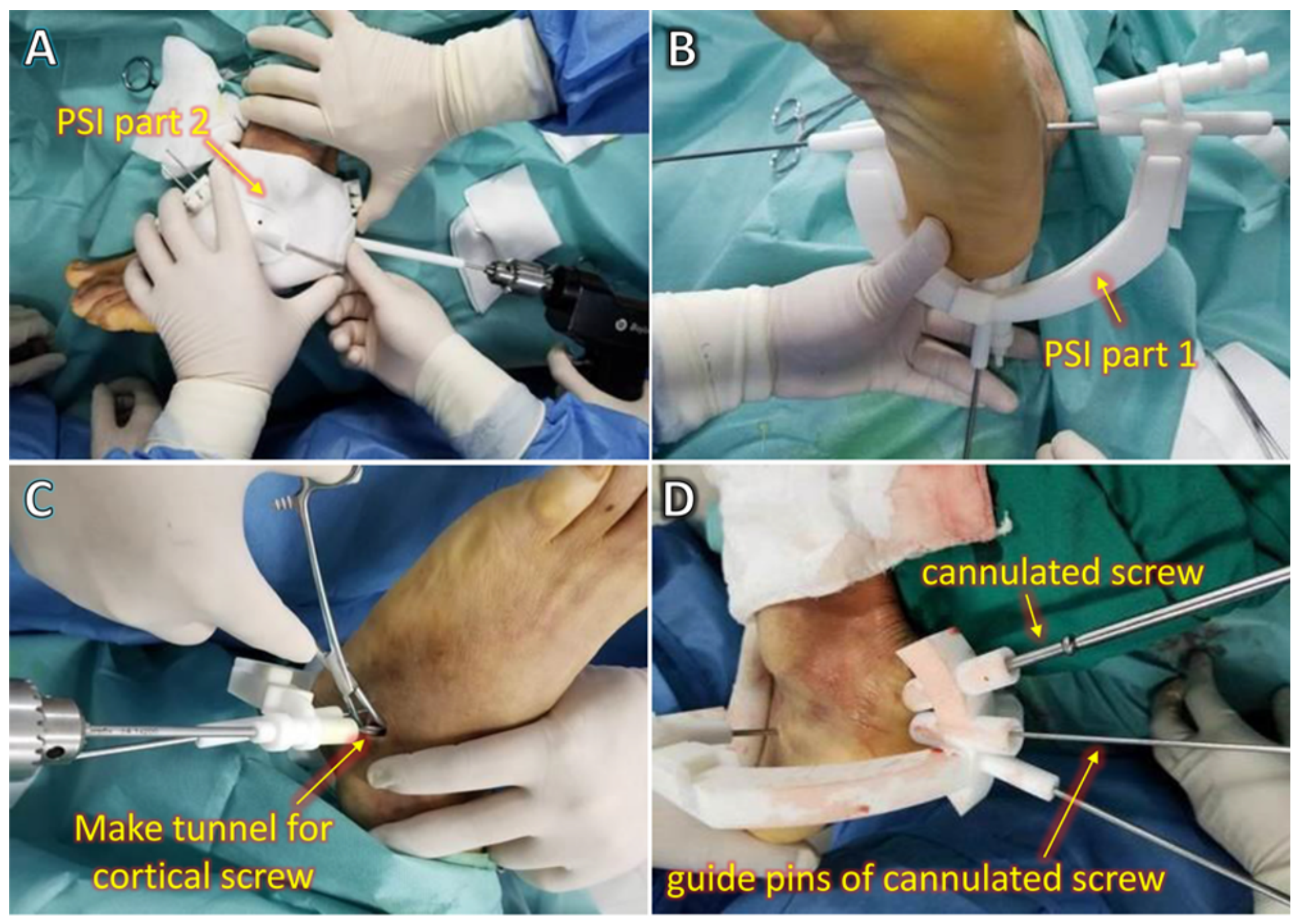

\section{Figure 5}

An example of application of the PSI during the patient's surgery. (A) PSI Part 1 was assembled, and 3 Schanz pins were used to connect the calcaneus fragments. (B) Guided by PSI Part 2, anatomical reduction was achieved. (C) Also guided by PSI Part 2, a lateral screw was inserted to fix the subtalar articular surface, and (D) two posterior cannulated screws were inserted to fix the main body of the calcaneus axially. 


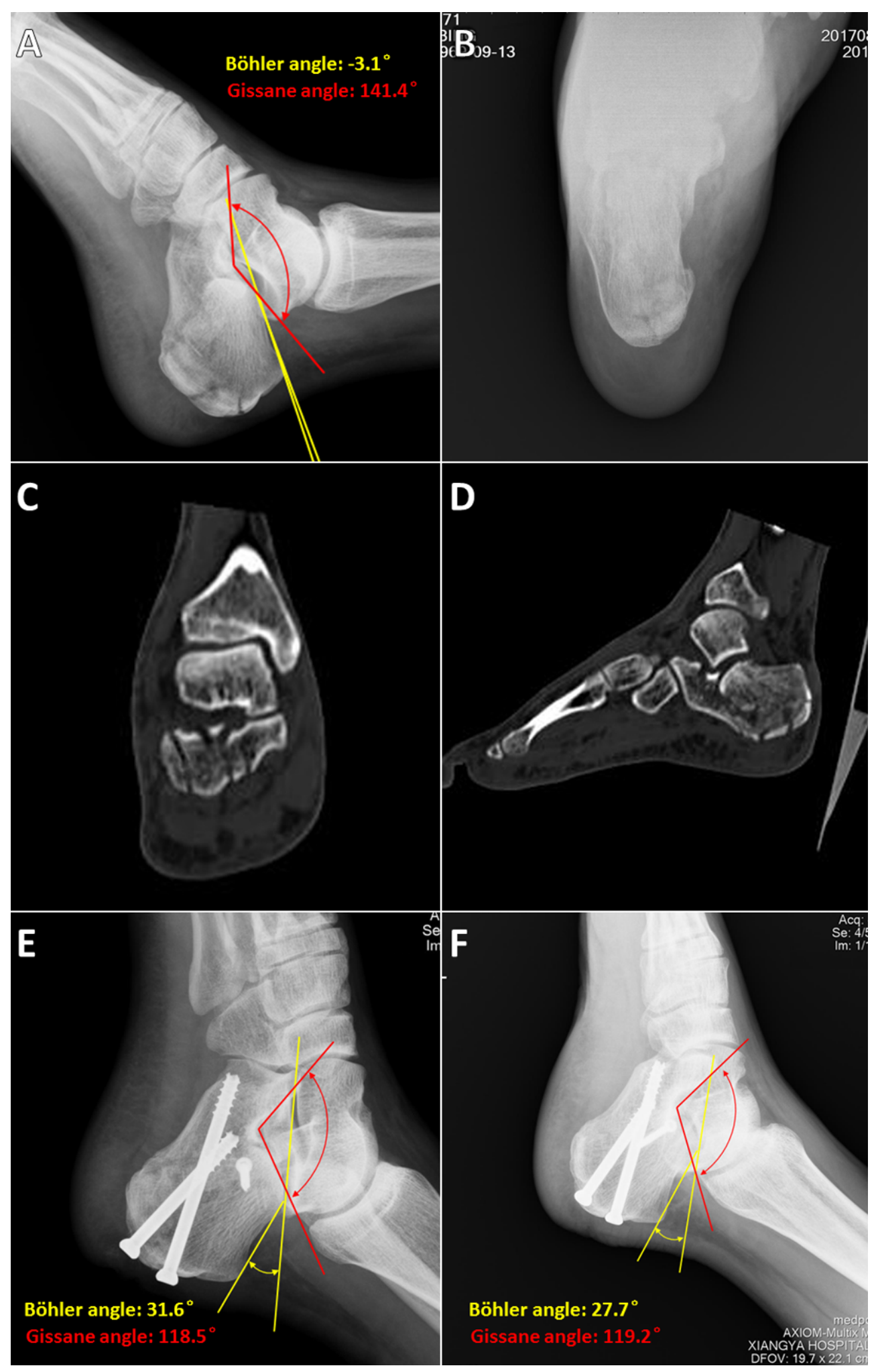

\section{Figure 7}

Imaging examination and evaluation (a typical case as an example, male, 47Y, left Sanders Type IV fresh calcaneal fracture): (A) An intra-articular fracture of the calcaneus is clearly depicted. The Böhler angle was $-3.1^{\circ}$, and the Gissane angle was $141.4^{\circ}$. (B) Axial view shows a varus deformity of the calcaneus. $(C, D)$ Coronal and sagittal views of the calcaneus computed tomography show irregular facet of the subtalar joint, and this is a Sanders type IV calcaneus fracture. (E) A lateral radiograph obtained 2 days 
after the surgery confirmed anatomical reduction of the calcaneus and stable fixation. The Böhler angle was $31.6^{\circ}$, and the Gissane angle was $118.5^{\circ}$. (F) A lateral radiograph obtained at approximately 12 months verified the anatomical reduction and stable fixation, with a Böhler angle of $27.7^{\circ}$ and Gissane angle of $119.2^{\circ}$.

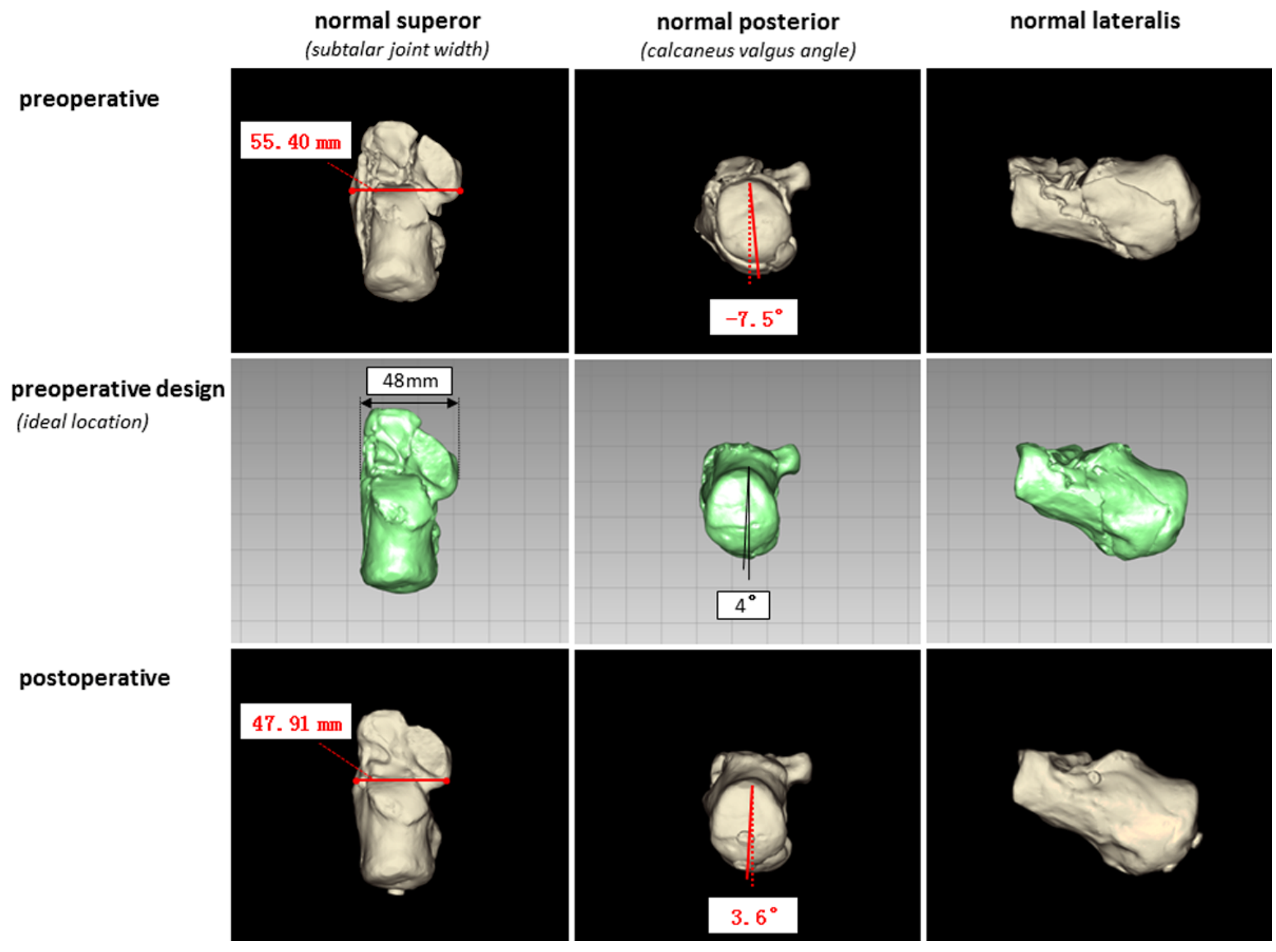

\section{Figure 9}

The CT data of preoperative actual measurement, preoperative-plan and postoperative were imported into the computer and obtain "reconstructed 3D digital model". We measured subtalar joint width in the position of sustentaculum by normal superor perspective, and measured calcaneus valgus angle by normal posterior perspective. 

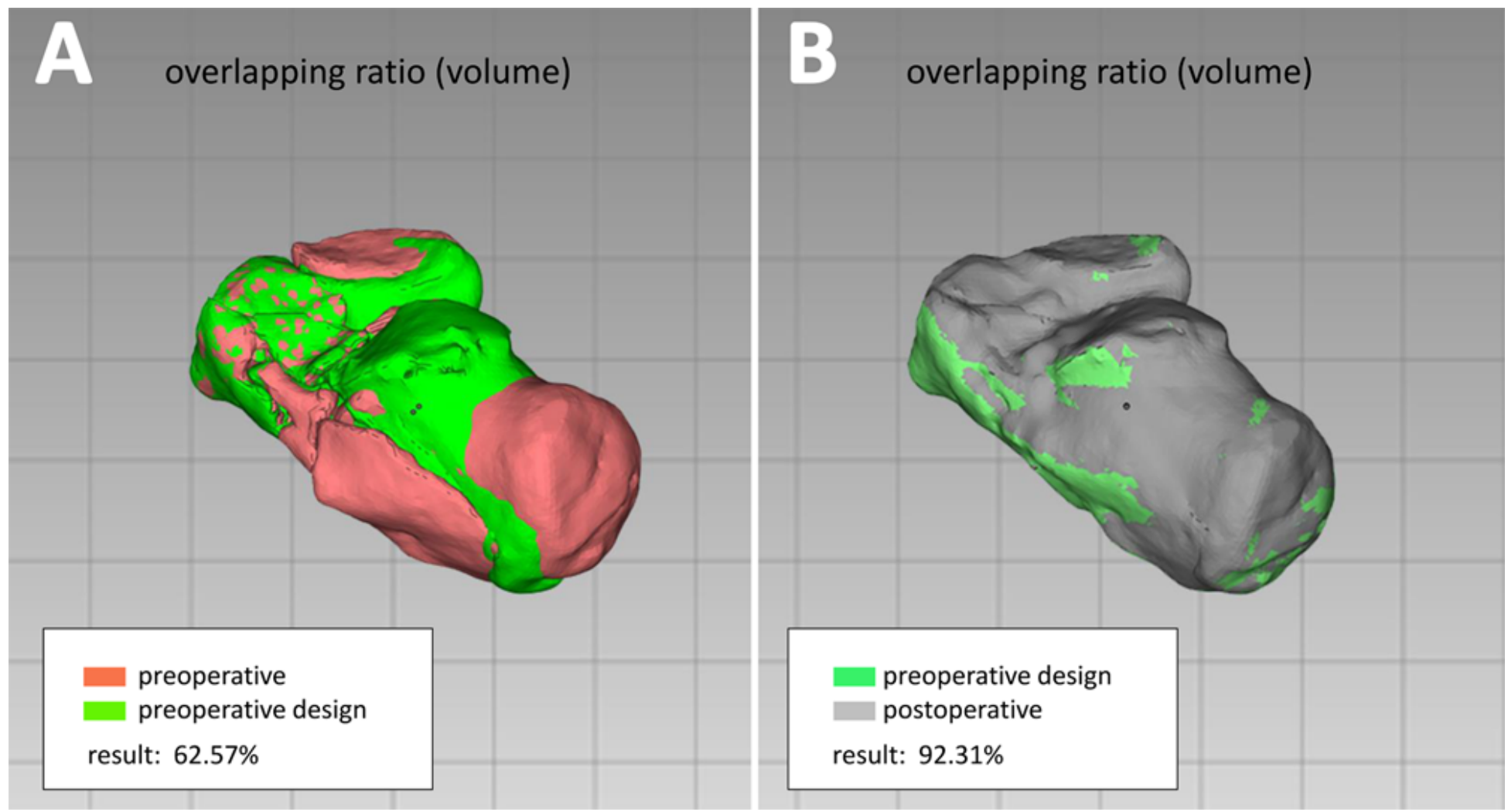

\section{Figure 11}

The calcaneal volume overlapping ratio. (A) The calcaneal volume overlapping ratio of preoperative with preoperative design. (B) The calcaneal volume overlapping ratio of preoperative design with postoperative which internal fixation has been excluded.
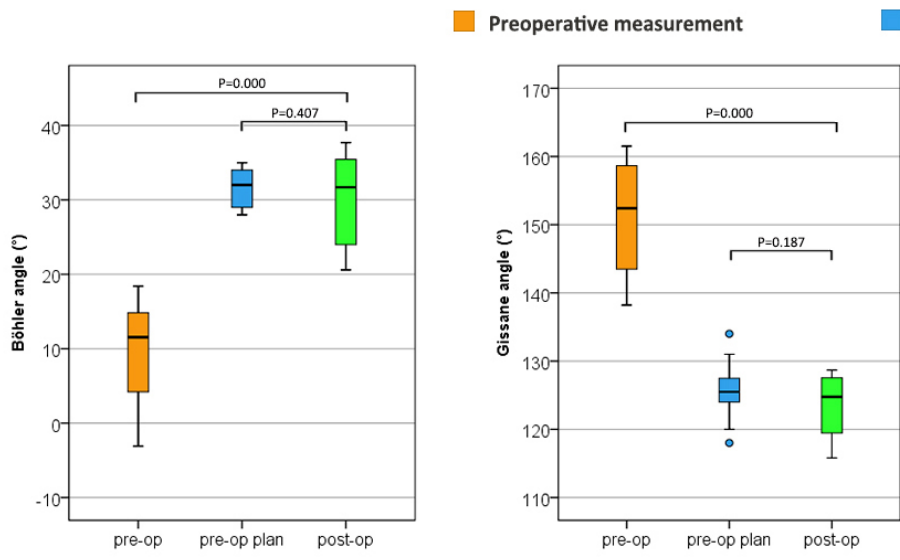

Preoperative plan

$\square$ Postoperative measurement
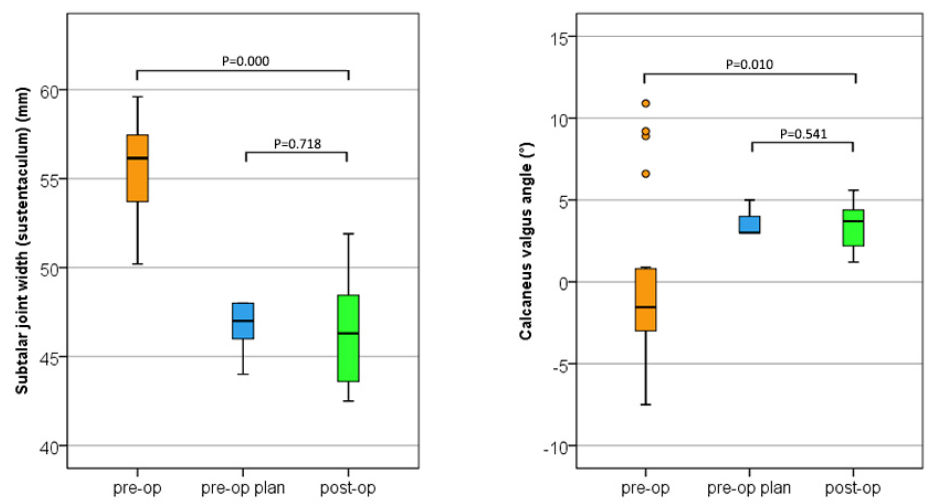

\section{Figure 13}

Box diagrams of Böhler angle, Gissane angle, subtalar joint width and calcaneus valgus angle. From these box diagrams, we can found that all data of preoperative plan were not significant different from the postoperative, all data of preoperative actual measurement were significant different from the postoperative. 


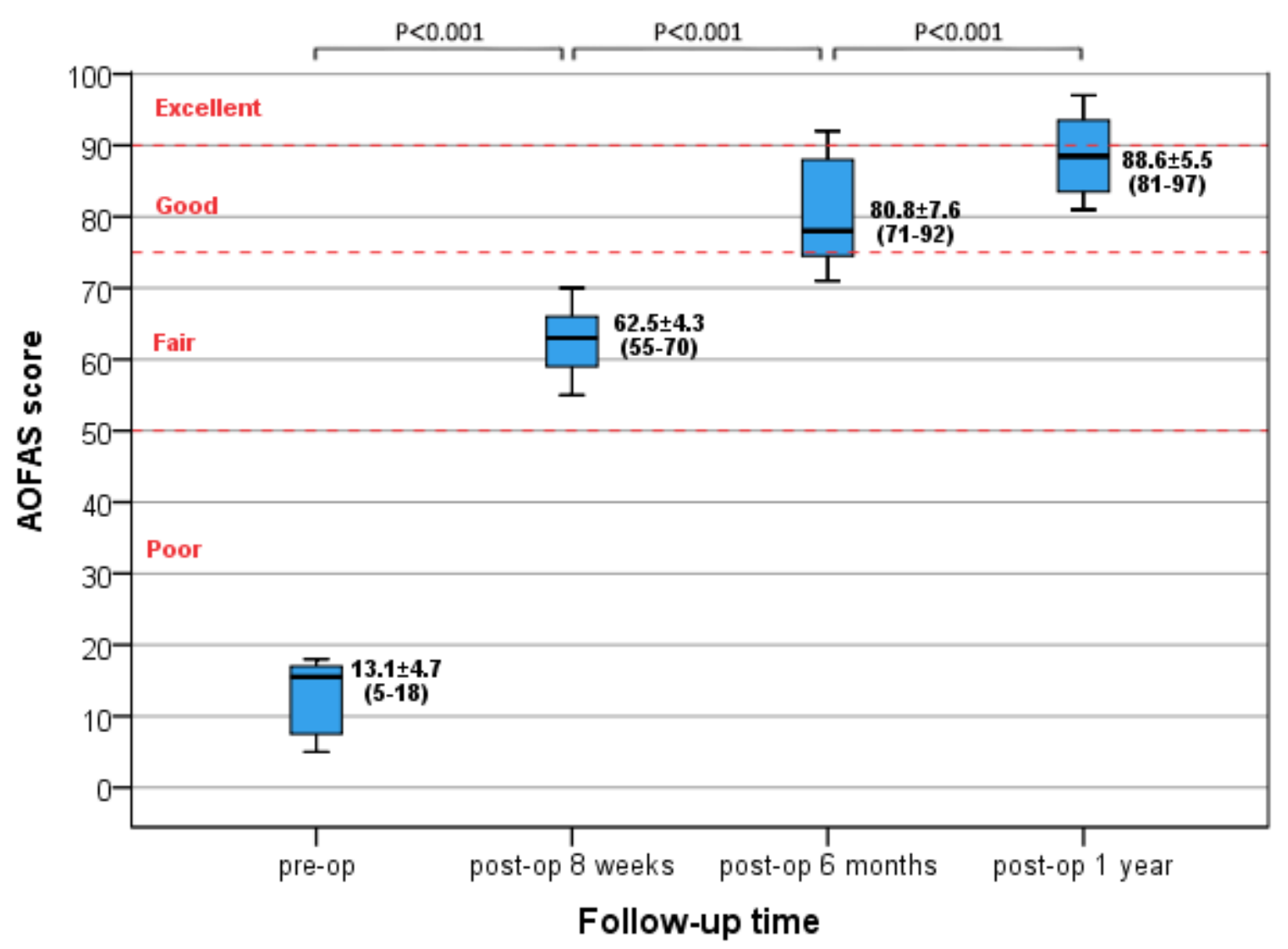

Figure 15

Box diagrams of AOFAS score of patients at each time point. We can found that the AOFAS score of all patients gradually increased as time goes on, and difference of the AOFAS scores between all time-points were significant.

\section{Supplementary Files}

This is a list of supplementary files associated with this preprint. Click to download.

- Video1.ThePSIdesignandsurgicalapplicationforexample.mp4

- Video1.ThePSIdesignandsurgicalapplicationforexample.mp4 